\title{
Les politiques indignés sur les sites internet
}

\author{
The indignant politicians on the Internet
}

\author{
Elżbieta Pachocińska \\ Université de Varsovie \\ e.pachocinska@uw.edu.pl
}

\begin{abstract}
Indignation in the political discourse has a long rhetoric tradition because of its moral aspect. This article focuses on the discursive construction of this emotion by French politicians in their communiqué posted on officials websites and on their twitter. It will be asked (1) how expression of indignation affects discursive structure, (2) how it can be inferred referring to our stereotypes, (3) how the emotional involvement contributes to positive ethos of politicians. Our analysis is based on the study of emotion in discourse proposed by Ch. Plantin (2011), the notion of subjectivity in language (C. Kerbrat-Orecchioni 1980) and the concept of ethos D. Maingueneau (1999).
\end{abstract}

Keywords: ostensive indignation, indignation inferred, political discourse

\section{INTRODUCTION}

L'indignation en discours politique a une longue tradition rhétorique. Liée au discours épidictique, à l'acte de blâmer, de dévaloriser un adversaire politique, elle motive la réaction critique. Depuis ce temps où la parole était prononcée sur la place publique, les politiques disposent d'un nouveau support-d'internet permettant de faire circuler leur parole dans l'espace public.

L'article se concentre sur la mise en scène discursive de l'indignation par les politiques dans les communiqués (de presse) postés sur leurs sites officiels et dans les twitts entre 2013-2014. Vu le caractère spécifique de ce genre discursif, i.e. le communiqué est un texte de réaction aux événements en cours, posté par les acteurs politiques pour faire connaître leurs opinions et les faire circuler dans les médias. Les exemples choisis pour l'analyse représentent des situations typiques où les politiques expriment leur indignation. Cela nous permettra de voir le rôle des facteurs pragmatiques dans l'interprétation de l'émotion. 
D'autres observations portent sur les façons de dire et de montrer l'indignation par des politiques qui visent ainsi à construire une image positive de soi dans l'espace public. Nous essaierons également de montrer de quelle façon l'engagement émotionnel du locuteur se projette sur la structure du discours.

Dans notre travail, nous nous appuyons sur la méthode d'analyse du discours émotionné proposée par Ch. Plantin (2011), pour classer le vocabulaire, nous nous référons à la notion de subjectivité en langue de C. Kerbrat-Orecchioni (1980), ainsi qu'aux recherches de D. Maingueneau (1999) concernant l'éthos dit et montré.

\section{SIGNIFICATION DE L'INDIGNATION}

Commençons par les définitions et les emplois du lexème "indignation » pour bien saisir sa particularité. C'est un «sentiment de colère et de révolte suscité par tout ce qui peut provoquer la réprobation et porter plus ou moins atteinte à la dignité de l'homme » (TLFi). Le comportement d'une personne indignée peut se manifester par les : cris et larmes. Pour décrire l'intensité de l'émotion, on parle d'une grande ou vive indignation, les motifs peuvent être qualifiés de justes et légitimes. Les verbes qui décrivent la façon de la communiquer sont les suivants : exprimer, manifester, partager l'indignation. Une situation, un comportement, des paroles de quelqu'un peuvent provoquer ou soulever l'indignation. Les dictionnaires mentionnent aussi l'indignation (générale), i.e. un mouvement de protestation auquel s'associent plusieurs personnes pour exprimer un tel sentiment. Les synonymes en usage : tollé (général); indignation du peuple; indignation publique (TLFi., LNPR) ${ }^{1}$. À la même famille appartiennent les lexèmes suivants : indigner (v. tr.), s'indigner (v. pron.), indigné (adj.), indigne (adj.), indignement (adv.), indignité (n.f.) ${ }^{2}$.

Ce rapide examen montre que l'indignation est définie comme un sentiment appartenant au champ conceptuel de la colère. Pourtant, il y a des différences entre les deux émotions. La colère est définie comme un "violent mécontentement accompagné d'agressivité ». Les synonymes en usage sont : courroux, emportement, exaspération, fureur, furie. Les signes extérieurs qui l'accompagnent sont d'ordre physique et physiologique : être rouge, blême de colère, bégayer, suffoquer, trembler, trépigner de colère. La personne qui parle avec

\footnotetext{
${ }^{1}$ Trésor de la langue française informatisé (TLFi), Le Nouveau Petit Robert (LNPR) 1993.

${ }^{2}$ Voir aussi Ch. Plantin (2012) : «Indignes, indignités, indignés : la construction argumentative de l'indignation ». Les discours en classe de français. Recherches. Revue de didactique et de pédagogie du français, 56, http://icar.univlyon2.fr/membres/CPlantin/documents/publis classees/35_2012c_Indign.pdf
} 
colère peut crier, injurier, jurer, pester. La famille du mot colère : coléreux (-euse), colérique (LNPR 1993).

Ces quelques observations montrent pourquoi les hommes politiques préfèrent s'indigner que se mettre en colère dans leurs discours publics. Certainement l'agressivité dans le comportement des politiques n'est pas bien vue par un large public, tandis que la personne indignée se présente comme motivée par un sentiment éthique et moral. Ces qualités de caractère sont particulièrement importantes pour les politiques qui tout au long de leur carrière construisent une image identitaire de soi (ethos) dans l'espace public.

\section{2. ÉMOTIONS EN ANALYSE DU DISCOURS}

Les recherches importantes sur le rôle des émotions dans le discours ont été menées par Christian Plantin qui dans son livre Les bonnes raisons des émotions. Principes et méthode pour l'étude du discours émotionné (2011) propose de faire une différence entre la communication émotionnelle et émotive. La première est à étudier par le psychologue, tandis que la deuxième par l'analyste du discours. La communication émotive se caractérise par l'usage stratégique et intentionnel de l'émotion en discours. Selon le chercheur, l'émotion se manifeste dans le discours de deux façons : directement, i.e. elle est déclarée, affichée, dans un " énoncé d'émotion » explicite ou elle est repérée indirectement. Dans ce dernier cas, l'énoncé d'émotion est inféré par des «signaux aval» de l'émotion, un comportement perceptible caractéristique d'une personne émotionnée. Un autre indice qui permet de reconstruire l'émotion exprimée indirectement se situe au niveau des formes de discours propres à introduire une classe spécifique d'émotion, appelées des «signaux amont» de l'émotion (Plantin, 2011: 141-144).

En général, d'autres propositions d'analyse des émotions en discours vont dans le même sens. Patrick Charaudeau (2000 : 140-141) constate que l'analyste du discours peut essayer d'étudier l'émotion de trois façons. Tout d'abord, dans la perspective d'effet visé produit par le processus discursif l'insérant dans une communication. Ensuite, par «le champ thématique », « un univers de pathémisation » qui représente « une certaine organisation des topiques (imaginaires socio-discursifs) susceptibles de produire un tel effet ». La troisième possibilité concerne les stratégies « de la mise en scène discursive à visée pathémisante ». Les marques du pathémique se voient aussi au niveau du vocabulaire, des mots déclencheurs d'émotion comme «assassinat, complot, victimes » ainsi que dans les énoncés de type : "Assez ! » prononcés dans un contexte particulier (Charaudeau, $2000: 139$ ). 
Selon lui, l'analyse du discours «a pour objet d'étude le langage en tant qu'il fait sens dans une relation d'échange, qu'il est lui-même signe de quelque chose qui n'est pas dans lui et dont il est pourtant porteur $\gg$ (Charaudeau, 2000 : 127). Bref, l'émotion peut être étudiée au niveau morphologique, lexical, syntaxique et pragmatique du discours. Les manifestations de la subjectivité : affectivité, évaluation et valorisation en langue sont étudiées par Catherine Kerbrat-Orecchioni (1980). Leur description pose parfois des problèmes parce que les traits sémantiques de subjectivité appelés par la chercheuse les «subjectivèmes" sont souvent contextuels donc instables. Cette dernière remarque concerne surtout les axiologiques «faire usage des axiologiques, c'est dans une certaine mesure parler de soi » constate C. Kerbrat-Orecchioni (1980: 82).

Toutes ces remarques nous amènent à une constatation que dans chaque culture, il existe des scripts émotionnels permettant d'inférer la nature de l'émotion à partir de l'usage des formes d'expression dans la communication. En même temps, ces connaissances permettent au locuteur d'influencer l'interlocuteur, de jouer sur les émotions de l'autre pour réaliser ses propres intentions en employant des mots dont il connaît la puissance émotionnelle sur le public. En un mot, avoir recours au pathos. Dans un contexte politique, qui est celui de notre analyse, nous avons souvent affaire à un affichage des émotions par les acteurs politiques soit par des formes directes soit par des verbalisations qui les signalent indirectement. Pourtant, il faut souligner que même des formes indirectes sont suffisamment pertinentes pour reconstruire l'émotion grâce aux facteurs pragmatiques et cognitifs.

Les émotions permettent aux politiques de se construire une image de soi, appelé dans l'analyse du discours « ethos prédiscursif et discursif ». Le premier renvoie à l'image préexistante du locuteur et le second à l'image qu'il se construit en discours. Dominique Maingueneau (1999) constate que l'ethos peut être explicitement dit « je suis ceci ou cela » ou montré, dans ce cas il est inféré à travers le discours. Mais : «La distinction entre ethos dit et montré s'inscrit aux extrêmes d'une ligne continue puisqu'il est impossible de définir une frontière tranchée entre le „dit” suggéré et le „montré” non explicite» (Maingueneau, 1999: 91). L'ethos se reconstruit à partir des indices au niveau macro et micro du discours que le destinataire peut interpréter selon ses stéréotypes culturels. Dans nos exemples, nous avons affaire à l'ethos discursif montré bien que les politiques expriment souvent directement leur indignation.

Nous commençons nos analyses par les situations où l'indignation en discours politique est explicitement désignée pour terminer par les formes et les contextes qui permettent de l'inférer. 


\section{3. ÉMOTION EXPLICITEMENT DÉSIGNÉE}

(1) Un politique présente sa réaction officielle à un événement tragique (un communiqué officiel sur le site du parti)

Jean-François Copé est profondément choqué et indigné par la violente attaque armée dont Libération a fait l'objet

Je suis profondément choqué et indigné par $^{3}$ la violente attaque armée dont Libération a fait l'objet ce lundi. Quelques jours après une tentative d'attaque similaire contre BFM, c'est bien la profession des journalistes qui semble être visée. C'est l'idée même de la Liberté qui semble prise pour cible. La liberté d'expression et d'information sans laquelle il n'y a pas de démocratie possible. Personne ne peut l'accepter. Le criminel doit être interpellé au plus vite et condamné avec la plus grande sévérité. Toute ma compassion va aussi vers le photographe grièvement blessé ainsi que sa famille et ses proches. Au nom de toute l'UMP, j'exprime ma solidarité totale envers la rédaction de Libération ainsi qu'à l'ensemble des journalistes. Personne ne doit céder à la violence ou à la peur : tout doit être fait pour que les journalistes puissent continuer à exercer librement leur métier, dans la plus grande sécurité, au service des Français.

Jean-François Copé, Président de l'UMP ${ }^{4}$

Le politique désigne ses émotions dans l'énoncé d'émotion d'une façon directe; il se dit "choqué et indigné ». Il emploie deux termes d'émotion pour exprimer avec force son état émotionnel. Le verbe choquer apporte le jugement sur l'événement, vu comme opposé à la bienséance, à la raison, etc. ${ }^{5}$ L'expression de l'indignation ajoute une dimension morale à la réaction du politique qui justifie son état émotionnel en introduisant le contexte de cet événement tragique. La constatation que cet attentat à main armée a porté atteinte aux valeurs de « la liberté de la presse » lui permet de légitimer sa prise de parole en tant qu'individu et représentant du parti. Le politique parle de la victime (le photographe), exprime sa compassion, sa solidarité « totale » envers la victime, sa famille ainsi qu'à la rédaction du journal où cet attentat a eu lieu. Il exige la condamnation sévère du criminel. Dans la dernière phrase: «Personne ne doit céder à la violence ou à la peur $[\ldots]$ ». Le politique veut se montrer catégorique et décidé dans son jugement de l'événement. Ce communiqué permet au politique de construire son ethos d'homme de principes et d'humanité. C'est un communiqué officiel posté sur le site du parti pour montrer que le politique et son parti ne sont pas indifférents à des situations où les valeurs sont basculées. La situation officielle influence la forme et la rhétorique

\footnotetext{
${ }^{3}$ Nous soulignons dans les textes de nos exemples.

${ }^{4}$ http://www.u-m-p.org/actualites/espace-presse/jean-francois-cope-est-profondement-choqueet-indigne-par-la-violente-attaque-armee-dont-liberation-a-fait-lobjet-, consulté le 29.02.2014, nous mettons les fragments du texte en gras dans tous les exemples.

${ }^{5}$ Le Nouveau Petit Robert (1993).
} 
de l'expression émotionnelle : la condamnation de l'acte terroriste, l'expression de la compassion, le recours aux valeurs républicaines. Le politique mise sur la description des événements pour produire des effets pathétiques.

(2) Un parti indigné présente une réaction officielle à un discours indigne (un communiqué officiel sur le site du parti)

Europe Écologie Les Verts apporte son entier soutien à ces manifestations et appelle toutes celles et ceux qui ont été indignés par les atteintes récentes au pacte républicain à exprimer de manière claire et déterminée leur opposition à ces discours de haine et d'exclusion. Ces mots, notamment ceux proférés à l'endroit de la Garde des Sceaux, Christiane Taubira, portent directement atteinte aux principes d'égalité et de fraternité qui fondent la République.

Pascal Durand, secrétaire national Jean-Philippe Magnen, Elise Lowy, porte-parole ${ }^{6}$

Dans le contexte des luttes politiques, l'expression de l'indignation par un parti politique est souvent accompagnée d'un soutien et d'appels à la manifestation des personnes indignées, ici par les " discours de haine et d'exclusion » qui circulent dans l'espace public visant aussi la ministre de la Justice. L'indignation est légitimée par l'apparition de ces discours attaquant les valeurs républicaines : l'égalité et la fraternité. Cette prise de position est l'occasion pour le parti politique de monter quels principes et valeurs lui sont chers.

Le communiqué suivant renvoie à un événement particulier, très médiatisé à l'époque, il représente aussi comme le précédent une réaction officielle aux discours jugés par le parti comme indignes.

(3) Suite aux propos tenus par François Hollande souhaitant que Léonarda revienne sans sa famille et à ceux de Manuel Valls dans le Journal du Dimanche de ce 20 octobre, considérant qu'il s'agit là d'un « acte de générosité », EELV tient à exprimer sa colère, son indignation et son incompréhension $[\ldots]$.

Elise LOWY, porte-parole ${ }^{7}$

Dans le communiqué, on désigne quels propos prononcés par les politiques du gouvernement ont provoqué cette réaction du parti. Pour montrer l'intensité de l'indignation, on affiche aussi la colère et l'incompréhension. L'emploi du mot colère est justifié par les propos jugés indignes, cette émotion perd sa connotation négative dans ce contexte, on la montre comme une juste colère. L'expression de l'incompréhension renvoie à une réaction d'étonnement aux

\footnotetext{
${ }^{6}$ http://eelv.fr/2013/11/22/appel-a-manifester-le-30-novembre-marchons-contre-le-racisme/, consulté le 15.12.2013.

${ }^{7}$ http://eelv.fr/2013/10/20/francois-hollande-et-manuel-valls-sur-leonarda-des-propos-inhuma ins-et- incomprehensibles/, consulté le 15.12.2013.
} 
discours d'un membre du gouvernement. Vu le contexte dans lequel ce communiqué a été posté, le parti montre sa solidarité avec la victime de cet événement.

Les acteurs politiques ne s'expriment pas uniquement sur les sites officiels, ils utilisent aussi d'autres possibilités que leur offre internet, les sites plus personnels leur permettant de réagir vite ce qui peut être interprété par le public comme une réaction sincère, venant tout droit du cœur.

(4) Un politique indigné réagit à un discours indigne (un message posté sur son compte Twitter)

Maire d'Angers, Vice-Président du Conseil Régional des Pays de la Loire - \#Angers \#PaysdelaLoire - @AimerAngers

Je suis indigné par tant de bêtise et d'homophobie face à l'affiche honteuse du \#subway St. Serge. \#angers ville de tolérance et d'égalité.

\section{Réactions des internautes}

@fbeatse on ne parlera donc pas de votre "élection" à la mairie d'\#Angers. Car là il y a vraiment de quoi s'indigner.

@fbeatse Je suis indigné qu'un homme public bafoue à ce point la liberté de penser, qui est pourtant base fondatrice de notre constitution ${ }^{8}$.

Twitter impose une forme courte du message, une énonciation directe, claire et facile à comprendre, il n'y a pas ici de place à la rhétorique officielle. On peut y trouver plus fréquemment un vocabulaire subjectif parce que les twitts sont souvent considérés comme les messages personnels des politiques où ils peuvent s'exprimer avec plus de franchise que sur les sites officiels. La lecture des twitts est orientée par les mots soulignés sur lesquels on peut cliquer pour reconstituer le contexte de l'événement. Par ce franc parler sur son compte Twitter, le politique se construit un ethos de proximité et de connivence avec les internautes. Ce style du politique est montré par le substantif évaluatif « la bêtise » et l'adjectif évaluatif et axiologique ${ }^{9}$ " honteuse » provoquant de vives réactions des usagers surtout de ceux qui ne partagent pas ses opinions. Toute cette spécificité de la communication interactive sur Twitter est visible dans nos exemples. Ici, des internautes reprennent l'énoncé d'émotion du politique «Je suis indigné » pour lui parler de leur indignation motivée par son «élection », le mot est mis entre guillemets suggérant ainsi l'accusation de malhonnêteté. Le deuxième twitt exprime l'indignation d'un internaute provoquée justement par l'emploi de l'énoncé d'émotion par le politique en réaction aux propos homophobes. Selon l'internaute, le politique montre ainsi le manque de respect

\footnotetext{
${ }^{8}$ https://twitter.com/fbeatse/status/302548594349703168, consulté le 10.01.2014.

${ }^{9}$ Pour plus de détails voir C. Kerbrat-Orecchioni (1980 : 73-100).
} 
à la liberté d'opinion dans l'espace public en exprimant son indignation. L'internaute suggère de cette façon qu'il attend du politique à ne pas exprimer ses opinions personnelles dans les affaires publiques.

\section{4. ÉMOTION INFÉRÉE}

(5) Une réaction officielle à un discours indigne (un communiqué sur le site du parti)

M. Copé, qui n'en est plus à un mensonge ou à une manipulation près, s'autorise en toute indécence à accuser des magistrats indépendants de mener un " espionnage politique » de M. Sarkozy. Mais M. Copé ne parviendra pas à tromper les Français ! [...].

La manœuvre de M. Copé est grossière. [...], l'UMP porte gravement atteinte à la République. [...].

Qu'il laisse les juges travailler, qu'il laisse les enquêtes se poursuivre jusqu'à leur terme, qu'il laisse tranquilles des institutions que les siens ont considérablement malmenées pendant les cinq années où Nicolas Sarkozy était à l’Elysée. [...].

Harlem Désir, Premier secrétaire du Parti socialiste ${ }^{10}$

Ce communiqué témoigne d'une grande tension émotionnelle, il vise à produire l'effet d'une vive indignation ressentie par le politique. L'émotion est visible au niveau syntaxique par les points d'exclamation, le type de phrases impératives "Qu'il laisse... », les répétitions syntaxiques de mêmes structures illustrent le ton et la vocalité du texte. Le vocabulaire est évaluatif «la manœuvre grossière », "c'est un acte de dénonciation des mensonges, des manipulations dirigé vers l'adversaire politique ». L'opinion que «l'UMP porte gravement atteinte à la République » renvoie directement aux valeurs. Par cette façon de nommer et catégoriser le discours de son adversaire, le politique veut produire l'effet d'une forte indignation voire la colère.

Dans le contexte des luttes politiques intérieures, les adversaires s'attaquent sans merci. Le communiqué suivant représente un acte d'accusation du mensonge électoral présenté comme une action consciente pour tromper les Français.

(6) Une réaction officielle à un discours indigne (un communiqué sur le site du parti)

60000 postes : le scandale du mensonge, la honte de l'escroquerie politique !

L'escroquerie électoraliste dont a fait preuve le Président Hollande pour être élu en 2012.

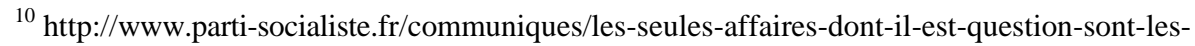
affaires-concernant-lump-0, consulté le 10.01.2014. 
[...] La décision du Gouvernement montre à quel point de cynisme électoral il a été amené, promettant tout, sans aucune intention d'accomplir quoi que ce soit.

[...] la liste est longue des promesses électoralistes que Monsieur Hollande n'a jamais eu la moindre intention de tenir. [...] rattrape et le confronte à sa démagogie, on ne peut que déplorer cette accumulation de manipulations.

[...]. Dans ce domaine, comme dans tant d'autres, Monsieur Hollande a délibérément menti et ses mensonges rejaillissent aujourd'hui et jettent le discrédit sur l'action politique.

Camille Bedin, Secrétaire générale adjointe de l'UMP ${ }^{11}$

On accuse le Président actuel, de faire des promesses sans avoir « la moindre intention de les tenir » tout cela, comme on le dit explicitement, pour gagner les élections présidentielles. On peut inférer ici une vive indignation voire la colère dont témoigne le niveau syntaxique du texte (les points d'exclamation), le lexique évaluatif et axiologique : le scandale, l'escroquerie, mentir, les mensonges, etc. dits dans un contexte politique précis. Quand le gouvernement déclare que certaines promesses électorales ne seront pas réalisées, c'est toujours une bonne occasion pour l'opposition, qui a perdu les élections, d'attaquer le gouvernement.

(7) Une réaction officielle visant la classe politique indigne (un communiqué de presse sur le site du parti)

La vie politique française prend une tournure déplorable. Multiples affaires Sarkozy, mensonges éhontés du gouvernement et notamment de la Garde des Sceaux : [...]

Il est inadmissible que le débat politique se vautre dans de telles bassesses, entre un parti devenu plaque tournante de toutes les affaires, et un autre en passe de devenir le syndicat des menteurs. [...]

Face à l'irresponsabilité de l'UMPS, Marine Le Pen appelle au sursaut citoyen. Loin de se détourner des urnes, les Français doivent profiter des élections municipales pour condamner sévèrement l'UMP et le PS, ces deux «culs-de-sac» de la politique française. $[\ldots]^{12}$

Ce communiqué dénonce la situation politique en France à la veille des élections municipales. On accuse les adversaires politiques des scandales, des mensonges, du manque d'un vrai débat dans l'espace public. Cette situation provoque un grand mécontentement des citoyens qui se projette sur les sondages prévoyant une grande abstention pendant les élections municipales. L'acte de dénonciation et d'accusation infère dans ce contexte politique l'indignation

${ }^{11}$ http://www.u-m-p.org/actualites/espace-presse/60-000-postes-le-scandale-du-mensonge-lahonte-de-lescroquerie-politique-105431103, consulté le 10.022014.

${ }^{12} \mathrm{http} / / /$ www.frontnational.com/2014/03/loin-du-marecage-umps-des-affaires-garder-le-capdes-vrais-problemes-des-francais/, consulté le 17.03.2014. 
dirigée vers le gouvernement et le parti adverse. Ce contexte politique permet à l'auteur du communiqué ici, la Présidente du FN de lancer un appel aux citoyens à voter contre la classe politique au pouvoir. De cette façon, elle construit son image et l'image de son parti soucieux des affaires intérieures du pays. Les évaluatifs et les axiologiques employés font référence implicitement à «l'affaire Buisson » qui scandalisait à l'époque l'opinion publique en France. La tournure impersonnelle « Il est inadmissible... » infère l'indignation, c'est un acte de dénonciation de la classe politique jugée indigne pour mener les affaires d'état.

(8) Une réaction officielle à un scandale politique (un communiqué du parti)

François Hollande doit s'expliquer sur ce qui s'apparente à un espionnage politique

Trop c'est trop. Cette affaire est désormais d'une gravité exceptionnelle : c'est l'Etat de droit qui est en cause.

Jean-François Copé demande au Président de la République de s'expliquer devant les Français sur cette affaire d'Etat qui s'apparente à un véritable espionnage politique. François Hollande se doit de garantir les libertés publiques au lieu de se comporter en digne héritier de François Mitterrand.

Dans une démocratie, il est inacceptable que les conversations entre un ancien Président de la République et son avocat puissent être écoutées sous des prétextes fallacieux tellement éloignés des motifs habituels de mise sur écoute comme le terrorisme, le grand banditisme, le trafic de drogue...

Dans une démocratie, il est inacceptable que les conversations des principaux responsables de l'opposition puissent faire l'objet d'écoutes et de rapports aux membres du Gouvernement.

Jean-François Copé ${ }^{13}$

L'idée du communiqué est explicitement présentée dans le titre, dès le début le contexte politique est introduit. Tout au long du texte se trouvent les indices montrant l'état émotionnel de l'auteur du communiqué : «Trop c'est trop...», les répétions syntaxiques «Dans une démocratie, il est inacceptable», les accusations prononcées sur un ton catégorique, le vocabulaire évaluatif «un véritable espionnage politique », "une affaire». Le politique dénonce les explications du gouvernement qu'il juge mensongères, l'accuse de ne pas respecter les valeurs fondamentales de l'État de droit. Le contexte décrit sert à justifier son indignation et sa colère. Pourtant la façon de l'exprimer par le politique montre son ethos d'un homme de principes.

${ }^{13} \mathrm{http} / / / \mathrm{www} . \mathrm{u}-\mathrm{m}-\mathrm{p}$. org/actualites/espace-presse/etre-ministre-de-la-republique-oblige-a-ladecence-a-la-juste-distance-a-levenement-au-strict-respect-des-institutions-nul-ne-peut-transigeravec-ces-exigences-105571303, consulté le 17.03.2014. 
(9) une réaction personnelle à la politique du gouvernement sur le Twitter et le blog Jean-Luc Mélenchon @JLMelenchon

\#Hollande, ami des patrons et président du \#chômage - Communiqué - http://bit.ly/ $\mathrm{N} 3 \mathrm{Hq} 8 \mathrm{~b}$ - \#France \#Emploi pic.twitter.com/eqFViX49od

communiqué du 26 février 2014

\section{Hollande, ami des patrons et président du chômage}

Le chômage atteint un nouveau record historique en France. Depuis l'élection de François Hollande, la hausse est continue : on compte 1000 chômeurs de plus par jour dans notre pays depuis mai 2012.

La moitié des chômeurs ne sont pas indemnisés. La misère s'accroît.

Mais depuis son élection, Hollande multiplie les milliards de cadeaux aux actionnaires. Le CAC 40 s'est engraissé de 34,62\% depuis mai 2012.

La seule courbe que François Hollande a réussi à améliorer c'est celle des profits. Hollande est l'ami de la finance et le président du chômage.

Trop c'est trop ! Nous ne sommes pas condamnés à subir. Avec la politique proposée par le Front de Gauche, le chômage baisserait. C'est ce que nous dirons dans les urnes le 23 mars et dans la rue le 12 avril prochain ${ }^{14}$.

Le message sur Twitter renvoie au billet posté sur le blog du politique où on peut trouver ce communiqué. Le politique décrit la situation sociale en France. Selon lui, le taux de chômage est extrêmement élevé, « la misère s'accroît ». Dans cette situation, le Président F. Hollande, issu du parti socialiste, «multiplie les milliards de cadeaux aux actionnaires ». Pour confirmer ses opinions, le politique cite les chiffres. La thématique et le style de son récit dans ce contexte visent, d'un côté, à exprimer son indignation, et de l'autre, à provoquer la même émotion du public.

\section{(10) Jean-Luc Mélenchon @JLMelenchon}

La honte. A Strasbourg, les socialistes, les verts et la droite votent pour l'espace ferroviaire libéral en \#Europe! \#UE_\#France_\#Rail

Le politique commente l'événement en le qualifiant de honte, cette ellipse infère l'énoncé : «Quelle honte ! » prononcé d'habitude dans la situation où on ressent une forte indignation. C'est l'exemple d'un mot, nous pouvons dire culturellement codé dans le sens qu' il désigne toujours l'indignation ou la colère selon les circonstances. Le motif de sa réaction est directement désigné étant donné que sur Twitter il n'y a pas de place pour argumenter.

(11) Jean-Luc Mélenchon@JLMelenchon ${ }^{15}$

Pacte de responsabilité : l'argent des contribuables arrosera (aussi) les actionnaires

\footnotetext{
${ }^{14} \mathrm{https}: / /$ twitter.com/JLMelenchon, consulté le 3.03.2014.

${ }^{15} \mathrm{https}: / /$ twitter.com/JLMelenchon, consulté le 17.03.2014.
} 
Le politique dénonce, selon lui, une des conséquences du «Pacte de responsabilité », un des projets politiques les plus importants du Président F. Hollande. Pour augmenter la tension émotionnelle, il emploie le mot dévalorisant « arroser » appartenant au registre familier. C'est la force illocutoire de l'acte de dénonciation exprimée dans ce twitt qui infère l'indignation.

(12) Force Républicaine @ForceRep 6 mar

Je demande au Président de la République d'arrêter de s'ingérer à tout bout de champ dans la vie privée des Français !" @FrancoisFillon ${ }^{16}$

C'est un appel qui fait référence aux mêmes événements politiques que nous avons déjà décrits (ex. 5, 8), il s'agit de l'affaire appelée « espionnage politique ». Cette réaction est présentée comme justifiée par les événements politiques portant atteinte aux valeurs démocratiques telles que la liberté des citoyens. L'appel au Président de la République lancé sur le compte Twitter du politique est plutôt symbolique et destiné à faire connaître l'opinion du politique dans l'espace public. Pour souligner la gravité de l'événement, il parle au nom des Français. Vu le contexte politique et la force illocutoire du message, on peut inférer qu'il est motivé par l'indignation.

(13) une réaction du parti politique sur son compte Twitter EÉLV@EELV

Corruption en France / EELV appelle à une révolution : l'indépendance du parquet http://bit.ly/1ip2rE4 \#eelv ${ }^{17}$

L'indignation peut être inférée à partir du vocabulaire et du contexte. L'émotion est montrée par les procédés d'amplification visibles dans les expressions hyperboliques. Ce twitt a la force illocutoire d'un acte d'accusation de corruption. Le dernier fragment suggère qu'il faut lutter pour «l'indépendance du parquet ».

\section{CONCLUSION}

Dans le discours politique, l'indignation se manifeste le plus souvent comme un acte de réaction ou de protestation. Elle donne l'occasion à la polémique, à la dénonciation et à l'accusation des adversaires politiques. La forme et la rhétorique de l'expression émotionnelle dépend de l'importance de l'événement, de l'image de soi que le politique veut se construire dans l'espace public. Même si l'acteur politique emploie le pronom « je », il reste toujours un représentant de

\footnotetext{
${ }^{16} \mathrm{https}: / /$ twitter.com/FrancoisFillon, consulté le 10.03.2014.

${ }^{17} \mathrm{https}: / /$ twitter.com/eel, consulté le 3.03.2014.
} 
son parti. Le fait de se dire indigné contribue à l'image positive du politique et du parti en créant un ethos d'humanité, de solidarité, etc. parce que les motifs présentés renvoient aux valeurs universelles sur lesquelles tout le monde est d'accord. L'acteur politique doit réagir par sa fonction même pour montrer qu'il n'est pas indifférent, quand on porte atteinte aux valeurs morales et démocratiques.

L'expression de l'émotion est souvent accompagnée d'appels à condamner un responsable, à appliquer des sanctions, à s'expliquer, etc. Le fait de parler et de montrer l'indignation dans l'espace public influence la forme du discours, tout d'abord, il faut justifier son état émotionnel ensuite, montrer son engagement pour se rendre crédible. Le politique affiche son émotion soit directement soit indirectement en ayant recours au vocabulaire qualifiant, aux structures syntaxiques précises (exclamations, questions), procédés rhétoriques (répétitions). Nous pouvons constater que l'expression de l'indignation est culturellement codée en langue, elle est dite et montrée par les formes spécifiques qui selon le contexte peuvent inférer aussi la colère.

L'impact d'internet est visible surtout dans la forme du message imposée par le type de site. Sur le blog ou Twitter le message est plus personnel, le vocabulaire plus axiologique, les formes plus directes. Les façons de dire et de montrer l'indignation dans le discours politique et surtout l'expression de l'intensité de l'émotion dépendent de l'événement qui fait l'objet de l'indignation. Quand cet événement présente dans une mauvaise lumière les adversaires politiques, on s'indigne d'une façon forte et violente indépendamment du site internet : officiel ou personnel. 


\section{BIBLIOGRAPHIE}

Charaudeau, P. (2000): «Une problématisation discursive de l'émotion. À propos des effets de pathémisation à la télévision ». In : Ch. Plantin, M. Doury, V. Traverso (dir.), Les émotions dans les interactions. Lyon : Presses Universitaires de Lyon, 125-155.

Kerbrat-Orecchioni, C. (1980): L'énonciation. De la subjectivité dans le langage. Paris: Armand Colin.

Le Nouveau Petit Robert. Dictionnaire alphabétique et analogique de la langue française (1993) : J. Rey-Debove et A. Rey (dir.). Paris : Dictionnaires Robert.

MAInguenEAU, D. (1999): «Ethos, scénographie, incorporation ». In : R. Amossy (dir.) : Images de soi dans le discours. La construction de l'ethos. Lausanne - Paris : Delchaux et Niestlé, $75-100$

Plantin, Ch. (2011): Les bonnes raisons des émotions. Principe et méthode pour l'analyse du discours émotionné. Bern : Peter Lang.

Plantin, CH. (2012): "Indignes, indignités, indignés: la construction argumentative de l'indignation ». In : Les discours en classe de français. Recherches. Revue de didactique et de pédagogie du français, 56. En ligne: http://icar.univ-lyon2.fr/membres/CPlantin/documents /publis_classees/35_2012c_Indign.pdf

Trésor de la langue française informatisé. http://atilf.fr

\section{Sitographie}

http://www.u-m-p.org/actualites/espace-presse/jean-francois-cope-est-profondement-choque-et-in digne-par-la-violente-attaque-armee-dont-liberation-a-fait-lobjet-, consulté le 29.02.2014.

http://eelv.fr/2013/11/22/appel-a-manifester-le-30-novembre-marchons-contre-le-racisme/, consulté le 15.12.2013.

http://eelv.fr/2013/10/20/francois-hollande-et-manuel-valls-sur-leonarda-des-propos-inhumains-etincomprehensibles/, consulté le 15.12.2013.

https://twitter.com/fbeatse/status/302548594349703168, consulté le 10.01.2014.

http://eelv.fr/2013/10/20/francois-hollande-et-manuel-valls-sur-leonarda-des-propos-inhumains-etincomprehensibles/, consulté le 15.12.2013.

http://www.parti-socialiste.fr/communiques/les-seules-affaires-dont-il-est-question-sont-les-affairesconcernant-lump-0, consulté le 10.01.2014.

http://www.u-m-p.org/actualites/espace-presse/60-000-postes-le-scandale-du-mensonge-la-hontede-lescroquerie-politique-105431103, consulté le 10.022014.

http://www.frontnational.com/2014/03/loin-du-marecage-umps-des-affaires-garder-le-cap-des-vraisproblemes-des-francais/, consulté le 17.03.2014.

http://www.u-m-p.org/actualites/espace-presse/etre-ministre-de-la-republique-oblige-a-la-decencea-la-juste-distance-a-levenement-au-strict-respect-des-institutions-nul-ne-peut-transiger-avecces-exigences-105571303, consulté le 17.03.2014.

https://twitter.com/JLMelenchon, consulté le 3.03.2014.

https://twitter.com/JLMelenchon, consulté le 17.03.2014.

https://twitter.com/FrancoisFillon, consulté le 10.03.2014.

https://twitter.com/eel, consulté le 3.03.2014. 